\title{
Quantum defect asymptotics at the critical charge: A study of the integrality conjecture
}

\author{
A. Sarsa \\ Departamento de Física, Campus de Rabanales Edif. C2, \\ Universidad de Córdoba, E-14071 Córdoba, Spain \\ fa1sarua@uco.es \\ and \\ J. Katriel* \\ Department of Chemistry, Technion - Israel Institute of Technology, Haifa, 32000 Israel \\ jkatriel@technion.ac.il
}

June 16, 2018

\begin{abstract}
A single $(n, \ell)$ electron outside an $(N-1)$-electron atomic core is bound as long as $Z>Z_{c}=N-1$. A conjecture is examined, according to which the quantum defect of the outermost electron satisfies $\lim _{Z \rightarrow Z_{c}} \delta_{n, \ell}(Z)=N_{\ell}$, where $N_{\ell}$ is the number of occupied or partially occupied orbitals with angular momentum quantum number $\ell$ within the $(N-1)$-electron core. Specifically, the $3 s$ quantum defect is inspected for the different occupancies of the $n=1$ and $n=2$ shells. The conjecture is found to hold in all the cases considered.
\end{abstract}

keywords: Quantum defect, critical charge, integrality conjecture.

\section{Introduction}

We consider an atomic system with $N$ electrons and nuclear charge $Z$. $(N-1)$ of the electrons form a core in some state $X$ and the remaining electron can be described as an $(n, \ell)$ electron, where the $(n, \ell)$ subshell is higher than any subshell occupied by core electrons. The $(N-1)$ electrons in the state $X$ and the additional $(n, \ell)$ electron are coupled into an $N$-electron state $Y$. The binding energy of the $(n, \ell)$-electron is defined as

$$
\epsilon_{n, \ell}=E_{N}(Y)-E_{N-1}(X) .
$$

${ }^{*}$ Corresponding author 
It can be written in the Rydberg-like form [1]

$$
\epsilon_{n, \ell}=-\frac{(Z-(N-1))^{2}}{2\left(n-\delta_{n, \ell}(Z)\right)^{2}},
$$

where $\delta_{n, \ell}(Z)$ is the quantum defect.

The quantum defect, that appears to be a mere reparameterization of the binding energy, satisfes the following properties

- $\delta_{n, \ell}(Z)$ is weakly dependent on $n$, fairly rapidly approaching an asymptotic value $\delta_{\infty, \ell}(Z)$, upon increase of $n$.

- Seaton's theorem [2]: $\pi \delta_{\infty, \ell}(Z)$ is equal to the zero energy $\ell$-wave phase-shift.

- $\delta_{n, \ell}(Z)$ decreases fairly rapidly with increasing $\ell$.

- For fixed $n$ and $\ell, \delta_{n, \ell}(Z)$ approaches zero as $Z$ rises (before relativistic effects take over).

\section{The integrality conjecture}

Inspection of experimental and computational data suggested the

Integrality conjecture: For fixed $n$ and $\ell$ the quantum defect of the outermost electron satisfies

$$
\lim _{Z \rightarrow(N-1)} \delta_{n, \ell}(Z)=N_{\ell},
$$

where $N_{\ell}$ is the number of occupied or partially occupied orbitals with angular momentum quantum number $\ell$ in the $(N-1)$-electron core.

The integrality conjecture can be rationalized by noting that when $Z$ is close to $(N-1)$ the outermost $(n, \ell)$ orbital becomes very diffuse. Relative to this orbital, the core becomes a point charge. The $(n, \ell)$ orbital approaches a hydrogen-like orbital with effective charge $Z-(N-1)$. Consistency of this asymptotic behaviour requires that the effective principal quantum number, hence the quantum defect, approaches some integer. The precise value of this integer can be inferred as follows. Recalling that the index that specifies the sequence of $\ell$-type orbitals is the radial quantum number $n_{r} \equiv n-\ell=1,2, \cdots$, it follows that $n_{r} \geq N_{\ell}+1$, or $n \geq \ell+N_{\ell}+1$. When $n=\ell+N_{\ell}+1$ the electron in this diffuse orbital is effectively in its ground state, that can be specified in terms of the effective principal quantum number $n_{e f f}=\ell+1$. Hence, the quantum defect is given by $n-n_{e f f}=N_{\ell}$.

The integrality conjecture is based on the following observations:

1. Ivanov, Bromley and Mitroy [3] investigated the $(1 s n s)^{3} S$ states of the He isoelectronic sequence. Translating their results into the conventions specified above, they established that the $n s$ quantum defects in all these states approach unity at $Z \rightarrow 1$.

2. For the $(1 s n p)^{1,3} P$ states Ivanov [4] established that the $n p$ quantum defect vanishes at the same limit. 
3. The integrality conjecture was proposed by Katriel, Puchalski and Pachucki [5], who studied the $(1 s 2 s)^{1} S$ state of the He isoelectronic sequence and the $\left(1 s^{2} 2 s\right)^{2} S$, the $\left(1 s^{2} 3 s\right)^{2} S$ and the $\left(1 s^{2} 2 p\right)^{2} P$ states of the $\mathrm{Li}$ isoelectronic sequence.

4. Further exploration of the integrality conjecture was reported by Katriel, Gaigalas and Puchalski [6], where this conjecture is referred to as the KPP conjecture. In that paper the experimentally determined quantum defects along several isoelectronic sequences are explored, demonstrating that in most cases they are consistent with the integrality conjecture, although, since they are restricted to integral $Z$ values, a precise extrapolation to the $Z \rightarrow Z_{c}$ limit is not feasible. The behaviour of the $2 p$ orbital in the ground state of the $\mathrm{B}$ isoelectronic sequence is more subtle. Extrapolation of the values of the quantum defect at integer $Z$ fails to suggest the asymptotic value that the integrality conjecture claims. A computation over fractional $Z$ values between the critical charge, $Z_{c}=4$, and $Z=5$, is required to account for the maximum of the quantum defect at $Z \sim 4.25$, followed by its rapid decrease upon further decrease of the nuclear charge. This computation confirms the integrality conjecture, that for this $2 p$ orbital predicts that $\lim _{Z \rightarrow 4} \delta_{2 p}=0$.

5. The $\left(1 s^{2} 2 s 3 s\right)^{1,3} S$ and the $\left(1 s^{2} 2 s 3 p\right)^{1,3} P$ states of the Be isoelectronic sequence were similarly investigated by Katriel et al. [7].

\section{Results}

The purpose of the present study is to provide a comprehensive examination of the integrality conjecture, considering all the systems in which the outermost orbital is $3 s$. The case $N_{\ell=0}=0$, in which no $1 s$ or $2 s$ orbital is occupied, is only represented by the hydrogen-like (one electron) atom, that trivially satisfies $\delta_{n \ell}(Z)=0$, hence the integrality conjecture, $\lim _{Z \rightarrow Z_{c}} \delta_{3 s}(Z)=0$. In the case $N_{\ell=0}=1$, e.g., $(1 s 3 s)^{1,3} S$ and $\left(1 s^{2} 3 s\right)^{2} S$ the integrality conjecture was confirmed in the references cited above. Here, we consider the configurations

- $Z_{c}=2:\left[(1 s 2 s)^{3} S 3 s\right]^{2,4} S$ and $\left[(1 s 2 s)^{1} S 3 s\right]^{2} S$.

- $Z_{c}=3:\left(1 s^{2} 2 s 3 s\right)^{1,3} S$ (cf. [7] as well).

- $Z_{c}=4:\left(1 s^{2} 2 s^{2} 3 s\right)^{2} S$.

- $Z_{c}=5:\left(1 s^{2} 2 s^{2} 2 p 3 s\right)^{1,3} P$.

- $Z_{c}=6$ : $\left[\left(1 s^{2} 2 s^{2} 2 p^{2}\right)^{3} P 3 s\right]^{2,4} P,\left[\left(1 s^{2} 2 s^{2} 2 p^{2}\right)^{1} D 3 s\right]^{2} D$, and $\left[\left(1 s^{2} 2 s^{2} 2 p^{2}\right)^{1} S 3 s\right]^{2} S$.

- $Z_{c}=10:\left(1 s^{2} 2 s^{2} 2 p^{6} 3 s\right)^{2} S$.

In Figure 1 we show quantum defects evaluated by using the NIST [8] experimental data. The $1 s^{2} 2 s^{2} 2 p^{3}, 1 s^{2} 2 s^{2} 2 p^{4}$ and $1 s^{2} 2 s^{2} 2 p^{5}$ quantum defects are not presented because the figure is already cluttered enough, but they fit within the space between curves XII and XIII. The curves are made to go through $\delta_{3 s}=2$ at $Z_{c}$, so this figure can claim to be consistent with the integrality conjecture, but certainly not to establish its validity. More convincing results can be obtained by computationally evaluating the quantum defect in the vicinity of the critical charge, allowing non-integral nuclear charges. Hartree-Fock energies were obtained, 
using the Froese Fischer code [9], for most of the systems specified above, as well as for the corresponding system with the $3 s$ electron removed. The quantum defects evaluated by using these energies are presented in Figure 2. The asymptotic trends suggested in Figure 1 are firmly established in Figure 2.

To avoid some technical complications we do not deal with the three-electron, three open-shells configuration $1 s 2 s 3 s$, as well as with the four electron $1 s^{2} 2 s 3 s^{1} S$ state, which is not the lowest ${ }^{1} S$ state of this system [10]. While the Hartree-Fock approximation does not include correlation, the asymptotic binding energy of the outermost electron can be safely assumed to be reliable, because correlation with the core becomes asymptotically negligible, and correlation within the core becomes asymptotically identical with that in the $(N-1)$-electron system.

\section{Discussion}

The most important result is that the asymptotic values of the quantum defects, at $Z \rightarrow Z_{c}$, where $Z_{c}$ is the number of electrons in the $n=1,2$ levels, is equal to 2 , which is the value of $N_{\ell=0}$ in all these systems. Whether the $2 s$ orbital is singly or doubly occupied, and what total spin it is coupled into, are asymptotically irrelevant.

The slopes of the curves can be interpreted as follows:

States corresponding to a common configuration: Consider the singlet and triplet $P$ states that correspond to the $1 s^{2} 2 s^{2} 2 p 3 s$ configuration. Since the triplet is lower in energy (by Hund's rule), the corresponding quantum defect should be higher (i.e., the effective quantum number is lower). In the configuration $1 s^{2} 2 s^{2} 2 p^{2} 3 s$ the order of the four levels is found to be [8] ${ }^{4} P<{ }^{2} P<{ }^{2} D<{ }^{2} S$. However, the quantum defects depend on the binding energies corresponding to three different $1 s^{2} 2 s^{2} 2 p^{2}$ states, namely ${ }^{3} P<{ }^{1} D<{ }^{1} S$. The two $P$ states correspond to the parent ${ }^{3} P$ state, so the quartet binding energy is higher than that of the doublet, yielding a higher quantum defect for the former. Since the ${ }^{2} D$ and the ${ }^{2} S$ binding energies are determined relative to the parent ${ }^{1} D$ and ${ }^{1} S$ states, respectively, the order of these binding energies, hence, the corresponding quantum defects, cannnot be simply deduced. The results suggest that these quantum defects are fairly close to one another, lying between those of the ${ }^{2} P$ and the ${ }^{4} P$.

States corresponding to distinct configurations: The overall trend suggests that the quantum defect increases, for a common deviation from the critical charge, with increasing number of electrons in the $n=1,2$ levels. This is probably due to the core contraction, which implies less penetration of the $3 s$ orbital into the core, making it more hydrogen-like.

Finally, we note that the insight into the asymptotic behaviour of the outermost orbital upon approaching the critical charge can be applied to the many atomic properties that depend on the shape of this outermost orbital. Work along these lines is underway.

Acknowledgement: One of the authors (AS) acknowledges partial support from the Spanish Dirección General de Investigación Cientfica y Técnica (DGICYT) and FEDER under contract FIS2015-69941-C2-2-P and from the Junta de Andalucía grant FQM378.

Declarations of interest: None. 


\section{References}

[1] T. F. Gallagher, Rydberg Atoms, Cambridge University Press, Cambridge 1994.

[2] M. J. Seaton, Rep. Prog. Phys. 46, 167 (1983).

[3] I. A. Ivanov, M. W. J. Bromley and J. Mitroy, Phys. Rev. A 66, 042507 (2002).

[4] I. A. Ivanov, Eur. Phys. J. D 27, 203 (2003).

[5] J. Katriel, M. Puchalski and K. Pachucki, Phys. Rev. A 86, 042508 (2012).

[6] J. Katriel, G. Gaigalas and M. Puchalski, J. Chem. Phys. 138, 224305 (2013).

[7] J. Katriel, J. P. Marques, P. Indelicato, A. M. Costa, M. C. Martins, J. P. Santos and F. Parente, Phys. Rev. A 90, 052519 (2014).

[8] Kramida, A., Ralchenko, Yu., Reader, J., and NIST ASD Team (2018). NIST Atomic Spectra Database (ver. 5.5.6), [Online].

Available: https://physics.nist.gov/asd [2018, May 24]. National Institute of Standards and Technology, Gaithersburg, MD.

[9] C. Froese Fischer, Comp. Phys. Comm. 43, 355 (1987).

[10] C. Froese, J. Chem. Phys. 47, 4010 (1967). 


\section{Figure captions}

Figure 1: Spectroscopic 3s quantum defects, based on NIST [8] data.

Figure 2: Computed $3 s$ quantum defects, based on Hartree-Fock binding energies. 